\title{
Disaggregation of Low-Resolution L-Band Radiometry Using C-Band Radar Data
}

\author{
C. Rüdiger, Senior Member, IEEE, C.-H. Su, D. Ryu, and W. Wagner, Senior Member, IEEE
}

\begin{abstract}
For Earth observation data to be useful for a wide range of land surface applications, a kilometer or finer resolution is required. Unfortunately, passive microwave observations at low microwave frequencies (1-10 GHz), already providing important information on soil moisture and vegetation dynamics, are generally only available at a resolution of tens of kilometers. This letter presents a new downscaling method relating L-band radiometer and C-band radar observations for downscaling purposes. The data were obtained from two extensive airborne field experiments across a $80000-\mathrm{km}^{2}$ catchment in south-eastern Australia and coinciding Envisat Advanced Synthetic Aperture Radar acquisitions, performed during the Austral summer and spring of 2010. The novel approach of this study is in the downscaling of coarse-scale emissivities as observed by the radiometer with a new interpretation of the change detection methodology for the radar signal to relate the spatiotemporal changes of those two types of observations at $1 \mathrm{~km}$. It is shown that, for most land surface conditions, a good spatial representation at high resolution is achieved, without considering land surface specific parameterizations, which is promising for using very high resolution radar data from the Sentinel-1 platform for downscaling of passive microwave data from current missions, such as National Aeronautics and Space Administration's Soil Moisture Active Passive and European Space Agency's SMOS.
\end{abstract}

Index Terms-Advanced Synthetic Aperture Radar (ASAR), downscaling, microwave radiometry, remote sensing, Sentinel-1, Soil Moisture Active Passive (SMAP).

\section{INTRODUCTION}

G LOBAL water resource management is an important aspect for meeting the growing demands for fresh water due to diverse types of anthropogenic pressure on this resource [1]. In addition, information on the hydric state of the land surface is important for other applications, such as environmental risk assessments (e.g., floods and fires), numerical weather prediction, and agriculture and ecosystem management. Satellite microwave observations have been shown to be sensitive to surface soil moisture variations [2]. To observe soil moisture glob-

Manuscript received September 2, 2015; revised December 21, 2015 and April 26, 2016; accepted June 6, 2016. This work made use of airborne data collected during the MoistureMap project, which was funded by the Australian Research Council (ARC DP 0879212).

C. Rüdiger is with the Department of Civil Engineering, Monash University, Clayton, Vic. 3800, Australia (e-mail: chris.rudiger@monash.edu).

C.-H. Su and D. Ryu are with the Department of Infrastructure Engineering, University of Melbourne, Melbourne, Vic. 3053, Australia.

W. Wagner is with the Department of Geodesy and Geoinformation, Technical University Vienna, 1040 Vienna, Austria.

Color versions of one or more of the figures in this paper are available online at http://ieeexplore.ieee.org.

Digital Object Identifier 10.1109/LGRS.2016.2583433 ally, a number of microwave satellite missions were designed to observe this land surface variable at a relatively high rate of every 2-3 days. In particular, the European Space Agency's (ESA) Soil Moisture and Ocean Salinity mission (SMOS, launched in November 2009 [3]), the National Aeronautics and Space Administration's Soil Moisture Active Passive (SMAP, launched in January 2015 [4]), and the Japan Aerospace Exploration Agency (JAXA) Global Climate Observation Mission for Water (GCOM-W1) satellite [5] with the second Advanced Scanning Microwave Radiometer (AMSR-2) onboard were specifically designed to observe soil moisture as one of their objectives. In addition, other sensors, such as the Advanced Scatterometer (ASCAT, launched onboard Metop-A (October 2006) and Metop-B (October 2012) [6]), allow deriving soil moisture from its active microwave backscatter observations. Similarly, high-resolution active microwave missions have also seen soil moisture products being developed, e.g., from ESA's Advanced Synthetic Aperture Radar (ASAR; launched onboard Envisat (March 2002) and operational 2004-2011 [7]), or JAXA's ALOS-PALSAR (ALOS-1 (in orbit 2006-2011 [8]). However, those products are often found to be relatively noisy and thus have a lower accuracy than the low-resolution missions [9].

For the purpose of numerical weather predictions, lowresolution, yet relatively accurate, observations are often sufficient. However, for water resource management applications (e.g., irrigation), as well as natural hazard predictions, a higher spatial resolution is required. To circumvent the main shortcoming of these microwave missions, i.e., mainly the relatively low spatial resolution of tens of kilometers in the passive and the high noise in the active data, the design of SMAP integrated both a radiometer and a radar [4], with a final soil moisture product of 9-km resolution, based on downscaling the passive soil moisture product using the active radar data to provide the spatial variation information [10], [11]. The approach of SMAP was to carry both active and passive sensors, allowing the use of temporally collocated data sets. The combination of radiometer and higher-resolution radar was unique, until the end of operations of the radar instrument in July 2015. The end of this part of the mission requires new methods of downscaling coarse resolution data.

As an alternative to downscaling using collocated microwave data sets, various disaggregation approaches based on optical imagery have been developed to obtain 1-km-resolution data for SMOS [12], [13]. The disadvantage of such methodologies is that optical and thermal data can be difficult to obtain due to frequent cloud cover, particularly in temperate regions. Consequently, merging existing high-resolution active microwave data sets with the low-resolution passive microwave observations needs to be considered as an alternative. 
The launch of ESA's Sentinel-1A satellite in 2014 (and its twin in 2016) opens up an excellent opportunity to develop weather-independent, high-resolution soil moisture products from low-resolution passive missions. Operating at C-band, Sentinel-1 delivers very high resolution data (of up to $5 \times 20 \mathrm{~m}$ ) with its final overpass repeat rate of 3-12 days depending on the region, which is comparable to the ASAR GM data availability of 3-8 days. In order to allow for the implementation of a robust downscaling methodology, the relationship between C-band backscatter and L-band radiometer data needs to be investigated first. The present paper uses data obtained from Envisat's ASAR to study its relationship with high-resolution passive L-band observations acquired during the Australian Airborne Cal/Val Experiments for SMOS (AACES [14]). While [15] already reported a good correlation between similar data sets over the central-Australian arid zone, transferability of the correlation to broader categories of terrain and surface conditions still needs to be investigated.

The regression-based merging methodology developed for SMAP in [10] is adapted here. Apart from only considering L-band emissions, the direct comparison of brightness temperature and backscatter is problematic because it fails to account for the significant influence of land surface temperatures on brightness temperatures, which is absent in backscatter observations. This problem was also identified in [16] where the relationship between active and passive observations were analysed for the Aquarius mission. As in [16], the regression parameters in this paper are derived from passive microwave emissivities and the change detection signal of the radar backscatter for a subset of the AACES-1 data. The disaggregation scheme is then evaluated using high-resolution airborne radiometer data acquired during AACES-2.

\section{DATA SETS}

\section{A. Airborne Radiometer Data}

The data sets used in this study are airborne L-band passive microwave observations collected during the AACES campaigns in south-eastern Australia [14]. The campaigns were conducted in the Austral summer (January-February 2010) and winter (September 2010) seasons. The Polarimetric L-band Microwave Radiometer (PLMR) was flown at an altitude of $10000 \mathrm{ft}(3000 \mathrm{~m})$ above ground, resulting in an average $3 \mathrm{~dB}$ footprint of $\sim 1 \mathrm{~km}$, and collecting data across most parts of the Murrumbidgee River catchment (Fig. 1). Operationally, the data are normalised to a $38^{\circ}$ reference incidence angle [17] to remove angular effects. The introduced additional error in the PLMR data due to this normalization procedure was shown to be in the order of $2.6 \mathrm{~K}$ for this data set [18], which will introduce uncertainties to the emissivities of less than $1 \%$ (for a reference land surface temperature of $280 \mathrm{~K}$ or more). During AACES-1, ten patches of $\sim 50 \mathrm{~km} \times 100 \mathrm{~km}$ were covered across a five-week period. The campaign started in very hot and dry conditions and finished with very wet $(\sim 200 \mathrm{~mm}$ precipitation over three days) and relatively cool conditions towards the end, due to a change in the continental weather patterns during this period, rather than spatially or topographically induced variations in the surface temperature. During the winter campaign, only the five central patches were covered, excluding the homogeneous/semi-arid western parts and the mountainous/

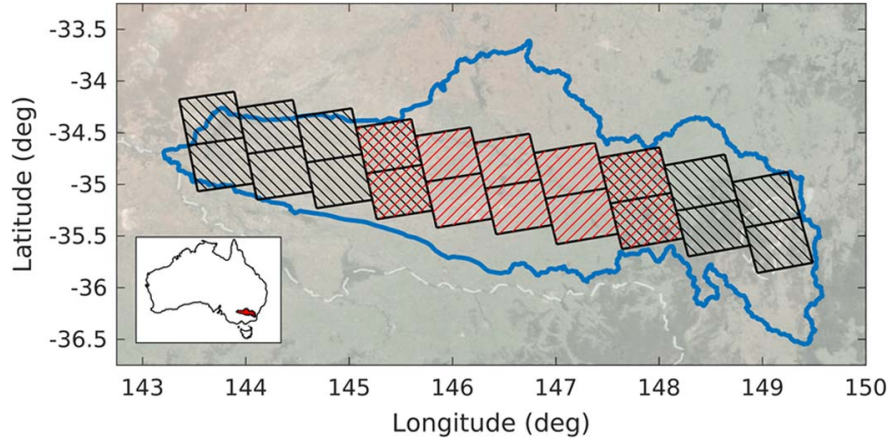

Fig. 1. Spatial overview of the individual flight patches. Black lines indicate the areas used for the regression model (only taken from the summer campaign), and red lines are for the validation areas (only taken from the winter campaign), refer to Table I for a detailed overview. The blue outline indicates the Murrumbidgee River catchment over a Landsat composite image.

urbanised eastern section of the catchment. The second campaign was marked by wet/cool conditions typical of a temperate winter and significantly higher levels of standing vegetation cover. More detailed information is presented in [14].

\section{B. Satellite Radar Data}

ASAR was operated on board the Envisat satellite from 2004 until 2011. Over Australia, SAR data were mainly acquired in its Global Mode (GM), resulting in overpasses every 3-8 days over the AACES patches within the Murrumbidgee River catchment. Those data are available at $1-\mathrm{km}$ resolution and are normalised to a $30^{\circ}$ reference incidence angle and the horizontally polarized $(\mathrm{HH})$ data were chosen for this study [19], as the ASAR GM data were generally provided in that polarization [7]. As the AACES campaigns were not designed to temporally collocate flights with ASAR, the closest overpass time to the PLMR acquisitions was chosen. In case of significant precipitation events, the closest date without precipitation interference was chosen.

\section{Methodology}

\section{A. Data Preprocessing}

The ultimate goal of this proof-of-concept study is the downscaling of SMOS and SMAP passive brightness temperature data, which are provided at incidence angles of $42.5^{\circ}$ and $40^{\circ}$, respectively, for their gridded higher level products. To study the possibility of a simple and directly applicable downscaling approach, both PLMR and ASAR data were not further corrected for their respective incidence angles, with the PLMR data being assumed to be representative of the SMOS/SMAP data when upscaled to $50 \times 50 \mathrm{~km}^{2}$. A full description of the operational PLMR processing chain (including correction for temperature drifts during the flights) is found in [17]. All corrections were done at the native resolutions, i.e., $500 \mathrm{~m}$ for ASAR and $1 \mathrm{~km}$ for PLMR. In order to reduce the noise levels, in particular the speckle noise in the ASAR observations, both data sets were aggregated to $2 \mathrm{~km}$ on a regular reference grid, using linear averaging with a simple nearest-neighbour approach. An aggregation to $4 \mathrm{~km}$ as suggested by [7] would have meant losing too much valuable spatial information. 


\section{B. Downscaling Approach}

To facilitate the transferability of the presented approach the approach developed for SMAP and presented in [10] and [11] was adapted, where

$$
T b_{p}\left(F_{j}\right)=T b_{p}(C)+\beta(C) \times\left[\sigma_{p p}\left(F_{j}\right)-\sigma_{p p}(C)\right]
$$

with $C$ and $F$ indicate coarse $(50 \mathrm{~km})$ and fine $(2 \mathrm{~km})$ resolution data; $T b_{p}\left(F_{j}\right)$ is the downscaled brightness temperature for a high-resolution pixel at location $j ; T b_{p}(C)$ is the observed brightness temperature at the coarse scale; $\beta(C)$ is the regression slope between active and passive observations at the coarse scale; $\sigma_{p p}\left(F_{j}\right)$ the radar backscatter for the high-resolution pixel at $j$; and $\sigma_{p p}(C)$ the aggregated backscatter data, upscaled to the resolution of the low-resolution radiometer $C$. The subscript $p$ refers to the polarization. Reference [11] also introduced a variable to implicitly consider vegetation conditions, through the use of the cross-polarised data of SMAP. As ASAR GM only rarely provided coinciding cross-polarised data, and Sentinel-1 is likely to only collect full acquisitions across selected regions of the world, it was necessary to employ the original algorithm of [10] to be globally applicable.

Other than in [20], the parameters here were developed for data mainly collected over patches separate to those chosen for the validation. Here, several changes were made to the SMAP approach: i) the development of the regression parameter is conducted at the $2-\mathrm{km}$ resolution instead of the coarse scale to obtain sufficient data points for the regression, and ii) the $\varepsilon_{p}$ and sensitivity $\Delta \sigma$ were used in place of $T b$ and $\sigma^{\circ}$. The reasons behind those changes are that brightness temperatures are directly affected by the effective surface temperatures and temperature drifts can be significant in this area (e.g., over $30 \mathrm{~K}$ as experienced during the AACES- 1 campaign). On the other hand, surface temperature drifts play an insignificant role in backscatter observations, while being more strongly affected by surface roughness. For the latter, ASAR data were corrected for their pixel specific dry reference, because of the non-negligent background bias due to temporally persistent roughness and vegetation that may not be seen in passive emissivities in the same way, but appear as a residual in the dry reference. Equation (1) is therefore reformulated to include the new definitions:

$$
\varepsilon_{p}\left(F_{j}\right)=\varepsilon_{p}(C)+\beta(C) \times\left[\Delta \sigma\left(F_{j}\right)-\Delta \sigma(C)\right]
$$

where

$$
\begin{aligned}
\varepsilon_{p}\left(F_{j}\right) & =\frac{T b_{p}\left(F_{j}\right)}{T_{s}} \\
\Delta \sigma\left(F_{j}\right) & =\sigma_{p}\left(F_{j}\right)-\sigma_{p, \text { dry }_{\text {ref }}}\left(F_{j}\right)
\end{aligned}
$$

and $T_{s}$ is the coarse-scale $\left(2 / 3 \times 1 / 2^{\circ}\right)$ modeled surface temperature taken from Modern Era Retrospective-Analysis for Research and Applications (MERRA) Land reanalysis [21]. A further assumption made is that $\beta\left(F_{j}\right)=\beta(C)$, which is similarly used in [10].

For the determination of the regression slope, the seven outer patches (1-4 and 8-10) of AACES-1 were chosen (Fig. 1) as they encompass the full range of soil moisture and general surface conditions (topography, vegetation, water bodies, etc.) found within the catchment. To validate the regression, data acquired over patches 4-8 during AACES- 2 in the following winter were used, making the validation data set both spatially

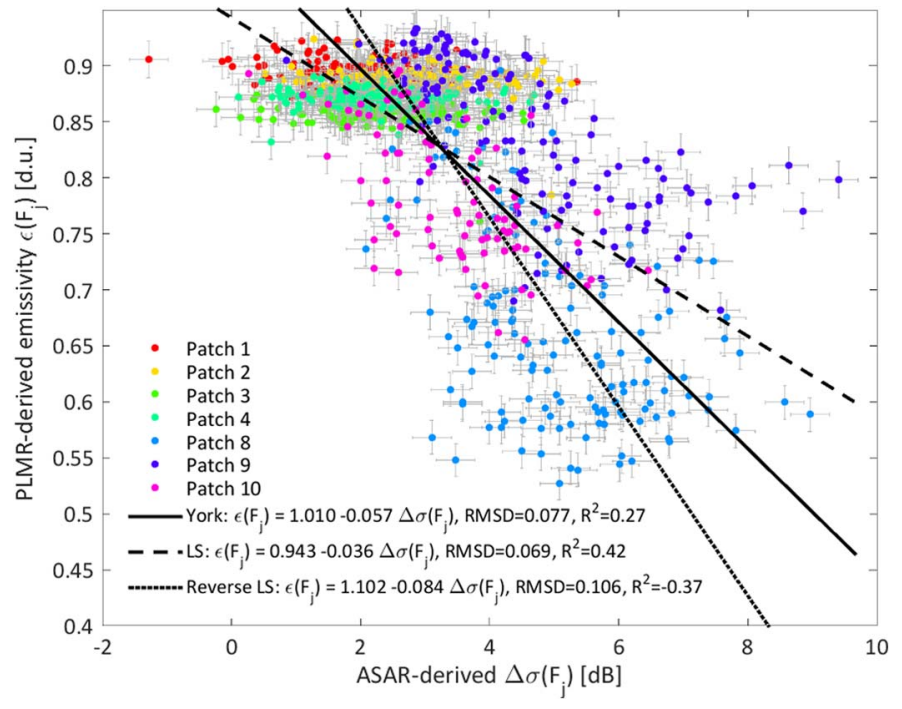

Fig. 2. Scatter plot including the three linear regression functions. The error bars show the assumed errors in the emissivity ( $4 \mathrm{~K}$ error in the brightness temperature $)$ and backscatter $(0.3 \mathrm{~dB})$ data. The MERRA soil temperature uncertainty was assumed to be $3 \mathrm{~K}$. For better visualization, only one in ten data points was plotted.

and temporally independent. While $T b_{p}\left(F_{j}\right)$ and $\Delta \sigma\left(F_{j}\right)$ were computed at 2-km resolution, $T b_{p}(C)$ and $\Delta \sigma(C)$ were the spatially aggregated brightness temperature and backscatter data, linearly averaged across each half patch or SMOS/SMAP footprint (i.e., $50 \mathrm{~km} \times 50 \mathrm{~km}$ ).

\section{Regression}

The regression parameter $\beta\left(F_{j}\right)$ was obtained through linear regression between $\varepsilon_{p}\left(F_{j}\right)$ and $\Delta \sigma\left(F_{j}\right)$, making use of two different approaches to determine a line of best fit, depending on the purpose of the analysis. First is the standard least-squares approach that yields a regression model for predicting $\varepsilon_{p}\left(F_{j}\right)$ from a given value of $\Delta \sigma\left(F_{j}\right)$. However, as the brightness temperature and backscatter observations are associated with measurement errors or uncertainties, this model can neither be used for making a reverse inference of $\Delta \sigma\left(F_{j}\right)$ based on $\varepsilon_{p}\left(F_{j}\right)$ nor transferred to other pairs of measuring systems with distinctive measurement errors. To make the reverse inference, the standard least-squares method can be performed with $\varepsilon_{p}\left(F_{j}\right)$ as the regressor. In contrast, the second approach based on an error-in-variable (EIV) model accounts for these measurement uncertainties. One such implementation is the York regression that regards (2) as an error-free model, but the regression is performed between the data pairs

$$
\begin{aligned}
& Y=\varepsilon_{p}\left(F_{j}\right)+E_{\varepsilon} \\
& X=\Delta \sigma\left(F_{j}\right)+E_{\sigma}
\end{aligned}
$$

with zero-mean additive Gaussian errors $E_{\varepsilon}$ and $E_{\sigma}$ explicitly added to allow for the interpretation of $\varepsilon_{p}$ and $\Delta \sigma$ as errorfree variables. York's line of best fit and the parameter $\beta\left(F_{j}\right)$ are then determined by minimizing its weighted distances from the data points (see (7) and (8), shown at the bottom of the next page). This study implements the York regression using known uncertainty estimates, namely, the observational errors in the backscatter and brightness temperature observations, 

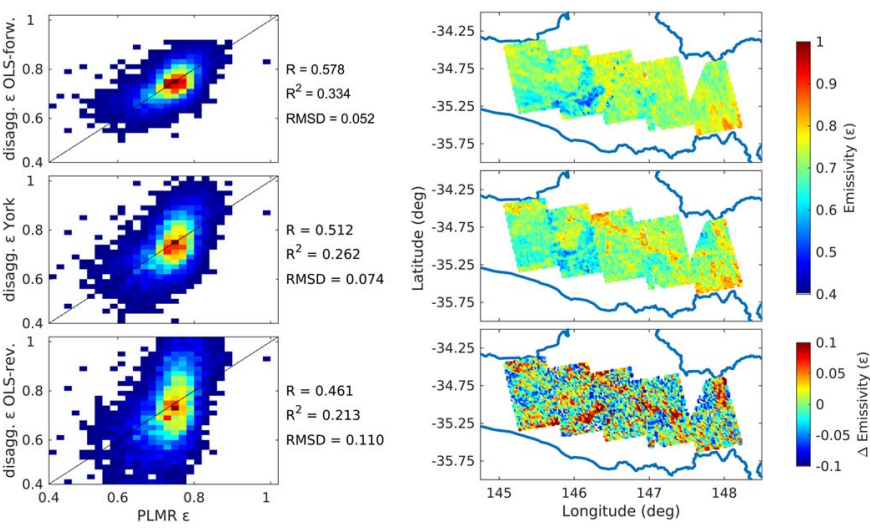

Fig. 3. Density plots showing the relationships between the downscaled (vertical axes) and original emissivities $(\varepsilon)$ derived from PLMR at $2 \mathrm{~km}$, based on the regression parameter of the three methods. The spatial plots show the PLMR (top) and downscaled (middle) emissivities using the OLS (forward) method and their difference (downscaled minus observed) for the five patches of AACES-2.

which were set to $0.3 \mathrm{~dB}$ [19] and $4 \mathrm{~K}$ [3], respectively, and $3 \mathrm{~K}$ for the land surface model soil temperature [22].

\section{RESUlTS AND Discussion}

Three distinctly different slope parameters are retrieved from the regression (Fig. 2), with the standard ordinary least-squares (OLS-forward) regression providing the best solution with an $R^{2}$ of 0.42 and an RMSD of 0.069 for the calibration patches. The OLS-forward method results in a relatively constrained range of emissivity values such that the ASAR end-member values match best. The constrained range of OLS-forward predictions can also be seen in Fig. 3. Using the aforementioned slope parameters for (2) yields emissivities for patches 4-8 during the winter campaign resulting in RMSDs in the emissivity estimation of 0.052 (OLS-forward), 0.074 (York), and 0.110 (OLS-reverse) when compared to their reference values. Using the standard configuration of the L-MEB radiative transfer model [23] for a bare soil with an average surface roughness and assumed effective land surface temperature of $280 \mathrm{~K}$, this uncertainty in the estimation of the emissivity translates into $0.06 \mathrm{~m}^{3} / \mathrm{m}^{3}$ soil moisture content for the standard least-square solution, which is comparable to or even better than other SMOS downscaling studies (e.g., [13]).

Fig. 3 shows the relationship of PLMR-derived and downscaled emissivities of the three regression methods (scatter plots), as well as the spatial representation for the forward least-squares method, leading to a downscaled product that is comparable to the original data (spatial plots). No bias is to be reported here as the presented downscaling method preserves the spatial mean, and the mean emissivities were calculated from PLMR data themselves.
Some artifacts, such as the significant overestimation of the emissivities by about 0.05 along the flood plains of the Murrumbidgee River (showing as a red band in the difference plots, running southeast to northwest in the downscaled emissivities), can be mainly attributed to the differences in the type of response between active and passive measurements. Other locations with significant overestimations of the emissivities are found in the south of the second patch, the east of the fourth patch, and the north-east of the last patch. In the first instance, this is due to a water-logged area (as evidenced by the very low observed emissivities), the city of Wagga Wagga, as well as an area with an increasingly steep terrain. All of these areas are not well covered by the regression method as the calibration data set largely did not include such areas. Interestingly, there is no clear pattern that can be associated with the forests within the catchment. As the systematic errors for the measurements due to land surface conditions are generalized for all vegetation types, such behavior was expected, and it suggests that a better handle on the observational error relationships needs to be developed to represent the diverse vegetation within a $50 \mathrm{~km} \times 50 \mathrm{~km}$ footprint. Moreover, for dry areas, the forward regression model (Fig. 2) is likely to work well for less vegetated areas than areas of high roughness, such as forests, where values with high- $\Delta \sigma$ values may lead to an overestimation of the emissivities.

Given that the data acquisitions were not coinciding, the low resolution of the land surface temperature field from MERRA-L, as well as a number of very strong precipitation events that occurred during AACES-1, this result is promising, given the good correlations for the forward least-square method of -0.649 (Pearson) and 0.528 (Spearman rank). It can now be argued that active C-band backscatter data may be used as a proxy to provide spatially high-resolution information on the soil moisture variability within a coarse-scale passive microwave pixel.

Some caveats have to be discussed at this point. First, the slope parameter in [10] assumes a linear and constant relationship between both brightness temperature and backscatter (or emissivity and sensitivity in the present study). This was already shown to be a source of error by [20], where it was suggested for the slope parameter to be derived individually for different vegetation covers. As such, this also partly contributes to the deficiency of the linear model to explain $58 \%$ of the variability (given $R^{2}=0.42$ ) in the data. Moreover, the relationship between L-band brightness temperatures and C-band backscatter is likely to be nonlinear [15]. To simulate the SMAP approach, this linearity was assumed here, but it will potentially have to be revised. The obvious nonlinear behavior is already seen for the dry and hot period (high emissivities and low sensitivities), where a small range of emissivity is found, while the sensitivity range is relatively large (being similar to wetter conditions). In addition, the acquisition of the data for patch 8 during AACES- 1 was preceded by precipitation with locally totaling $180 \mathrm{~mm}$ over two successive days, potentially biasing

$$
\begin{aligned}
& \lambda=\frac{\operatorname{var}\left(E_{\varepsilon}\right)}{\operatorname{var}\left(E_{\sigma}\right)} \\
& \beta=\frac{\operatorname{var}(Y)-\lambda \operatorname{var}(X)+\sqrt{[\operatorname{var}(Y)-\lambda \operatorname{var}(X)]^{2}+4 \lambda \operatorname{cov}(X, Y)^{2}}}{2 \operatorname{cov}(X, Y)}
\end{aligned}
$$


the regression. At 2-km resolution, ASAR GM data are also still relatively noisy [7], and the assumption of instrument and model errors may have to be revisited. However, Sentinel-1 has a significantly lower radiometric noise (or higher signalto-noise ratio) compared to ASAR, which is expected to reduce the currently apparent spatial noise. Finally, uncertainties in the accuracy of the precipitation events, the chosen soil temperature reference, and differences in dates of acquisition (patches 5-7 have the same ASAR data as a reference due to acquisitions closer to airborne data collections) may add additional levels of uncertainty. However, the latter may be irrelevant once data obtained from Sentinel-1 are used, allowing a more timely combination of the passive and active data sets.

\section{CONCLUSION}

This letter has presented a novel approach to synergistically use C-band SAR and L-band radiometer data to achieve a high-resolution (1-2 km) brightness temperature product (by means of downscaling the emissivity itself), irrespective of the weather conditions. The presented methodology uses the emissivities (radiometer) and sensitivities (radar) to remove surface temperature effects and to better retain the vegetation information in the downscaled product.

Making use of two field campaigns conducted in southeastern Australia, the downscaling approach resulted in emissivity uncertainties ranging from 0.052 to 0.110 , depending on the type of regression chosen, which translates to $0.06-0.12 \mathrm{~m}^{3} / \mathrm{m}^{3}$ errors in the soil moisture retrieval under standard conditions, which is comparable to other downscaling methods. This underlines the value of the Sentinel-1 mission in support of other microwave missions employing lower resolution radiometers, such as the recent SMOS, SMAP, and AMSR-2.

As this is a proof-of-concept study and a number of assumptions were made to simplify the complex relationship between active and passive microwave emissions across different bands, future work needs to revolve around understanding the influence of the instrument errors, as well as the distribution of the error structures themselves and the role of noncoinciding data acquisitions, errors due to spatial variability mismatching, and simple biases, or the likely effect of vegetation type on the downscaling algorithm. A better understanding of those errors or uncertainties will eventually allow to improve the regression model and to reduce the resulting soil moisture retrieval errors. In addition, different regression types should be tested taking into account the probable nonlinearity of those data sets, particularly during extreme conditions (dry or very wet/saline), and eventually Sentinel-1 data to apply this approach operationally using existing global soil moisture data sets.

\section{REFERENCES}

[1] B. Ostberg, J. Fabre, D. Ruelland, and A. Dezetter, "Historical reconstruction and 2050 projections of water demand under anthropogenic and climate changes in two contrasted Mediterranean catchments," J. Hydrol., vol. 522, pp. 684-696, Mar. 2015.

[2] T. J. Schmugge et al., "Remote sensing in hydrology," Adv. Water Res., vol. 25 , no. 8-12, pp. 1367-1385, 2002.
[3] Y. H. Kerr et al., "The SMOS mission: New tool for monitoring key elements of the global water cycle," Proc. IEEE, vol. 98, no. 5, pp. 666-687, May 2010.

[4] D. Entekhabi et al., "The Soil Moisture Active Passive (SMAP) mission," Proc. IEEE, vol. 98, no. 5, pp. 704-716, May 2010.

[5] H. Shimoda, "Overview of GCOM," in Proc. IEEE Int. Geosci. Remote Sens. Symp., 2013, pp. 827-830.

[6] W. Wagner et al., "The ASCAT soil moisture product: A review of its specifications, validation results, and emerging applications," Meteorol. Zeitschrift, vol. 22, no. 1, pp. 5-33, Feb. 2013.

[7] C. Pathe, W. Wagner, D. Sabel, M. Doubková, and J. B. Basara, "Using Envisat ASAR global mode data for surface soil moisture retrieval over Oklahoma, USA," IEEE Trans. Geosci. Remote Sens., vol. 47, no. 2, pp. $468-480$, Feb. 2009.

[8] M. Shimada and T. Otaki, "Generating continent-scale high-quality SAR mosaic datasets: Application to PALSAR data for global monitoring," IEEE J. Sel. Topics Appl. Earth Observ. Remote Sens., vol. 3, no. 4, pp. 637-656, Dec. 2010.

[9] I. Mladenova et al., "Validation of the ASAR global monitoring mode soil moisture product using the NAFE'05 data set," IEEE Trans. Geosci. Remote Sens., vol. 48, no. 6, pp. 2498-2508, Jun. 2010.

[10] N. N. Das, D. Entekhabi, and E. G. Njoku, "An algorithm for merging SMAP radiometer and radar data for high resolution soil moisture retrieval," IEEE Trans. Geosci. Remote Sens., vol. 49, no. 5, pp. 1504-1512, May 2011.

[11] N. N. Das et al., "Tests of the SMAP combined radar and radiometer algorithm using airborne field campaign observations and simulated data," IEEE Trans. Geosci. Remote Sens., vol. 52, no. 4, pp. 2018-2028, Apr. 2014.

[12] O. Merlin et al., "Self-calibrated evaporation-based disaggregation of SMOS soil moisture: An evaluation study at $3 \mathrm{~km}$ and $100 \mathrm{~m}$ resolution in Catalunya, Spain," Remote Sens. Environ., vol. 130, pp. 25-38, Mar. 2013.

[13] M. Piles et al., "A downscaling approach for SMOS land observations: Long-term evaluation of high resolution soil moisture maps over the Iberian Peninsula," IEEE J. Sel. Topics Appl. Earth Observ. Remote Sens., vol. 7, no. 9, pp. 3845-3857, Sep. 2014.

[14] S. Peischl et al., "The AACES field experiments: SMOS calibration and validation across the Murrumbidgee River catchment," Hydrol. Earth Sys. Sci., vol. 16, no. 6, pp. 1697-1708, Jun. 2012. [Online]. Available: 10. 5194/hess-16-1697-2012

[15] C. Rüdiger, M. Doubkova, J. R. Larsen, W. Wagner, and J. P. Walker, "Similarities between spaceborne active and airborne passive microwave observations at $1 \mathrm{~km}$ resolution," IEEE Geosci. Remote Sens. Lett., vol. 11, no. 12, pp. 2178-2182, Dec. 2014.

[16] X. Wu, J. P. Walker, N. Das, R. Panciera, and C. Rüdiger, "Evaluation of a brightness temperature downscaling algorithm using active and passive microwave observations," Remote Sens. Environ., vol. 155, pp. 210-221, 2014.

[17] M. Piles, K. A. McColl, D. Entekhabi, N. Das, and M. Pablos, "Sensitivity of Aquarius active and passive measurements temporal covariability to land surface characteristics," IEEE Trans. Geosci. Remote Sens., vol. 53, no. 8, pp. 4700-4711, Aug. 2015.

[18] M. Doubková et al., "How do spatial scale, noise, and reference data affect empirical estimates of error in ASAR-derived $1 \mathrm{~km}$ resolution soil moisture?" IEEE J. Sel. Topics Appl. Earth Observ. Remote Sens., vol. 7, no. 9, pp. 3880-3891, Sep. 2014.

[19] C. Rüdiger et al., "Towards vicarious calibration of microwave remote sensing satellites in arid environments," IEEE Trans. Geosci. Remote Sens., vol. 52, no. 3, pp. 1749-1760, Mar. 2014.

[20] N. Ye, J. P. Walker, and C. Rüdiger, "A cumulative distribution function method for normalizing variable-angle microwave observations," IEEE Trans. Geosci. Remote Sens., vol. 53, no. 7, pp. 3906-3916, Jul. 2015

[21] R. H. Reichle et al., "Assessment and enhancement of MERRA land surface hydrology estimates," J. Clim., vol. 24, no. 24, pp. 6322-6338, 2011.

[22] Y. Yi, J. S. Kimball, L. A. Jones, R. H. Reichle, and K. C. McDonald, "Evaluation of MERRA land surface estimates in preparation for the Soil Moisture Active Passive mission," J. Clim., vol. 24, pp. 3797-3816, 2011.

[23] J.-P. Wigneron et al., "L-band Microwave Emission of the Biosphere (L-MEB) model: Description and calibration against experimental data sets over crop fields," Remote Sens. Environ., vol. 107, no. 4, pp. 639-655, Apr. 2007. 